


\title{
COMMUTATIVITY THEOREMS FOR NONASSOCIATIVE RINGS WITH A FINITE DIVISION RING HOMOMORPHIC IMAGE
}

\author{
E. C. Johnsen, D. L. Outcalt, and Adil Yaqub
}

Wedderburn's Theorem, asserting that a finite associative division ring is necessarily commutative, has been extended to

Theorem 1. Let $R$ be a noncommutative Jordan ring of characteristic not 2 , and let $I$ be an ideal in $R$ such that $R / I$ is a finite division ring of characteristic $p>5$ with exactly $q$ elements. Suppose that (i) $I$ is commutative and every associator contained in the ideal generated by $I^{2}$ vanishes, and $\left(\right.$ ii) $x \equiv y(\bmod I)$ implies $x^{q}=y^{q}$ or both $x$ and $y$ commute with all elements of $I$. Then $R$ is commutative.

The object of this paper is to extend Theorem 1 in two directions. First we replace the assumption that $R$ is a noncommutative Jordan ring by the weaker assumption that $R$ is power-associative. Next we assume that $R$ is a flexible powerassociative ring but replace the hypothesis that every associator in the ideal generated by $I^{2}$ vanishes with the weaker assumption that $I$ is associative. In each case we drop the assumption that $R$ is of characteristic not 2 .

The proof of Theorem 1 appears in [2].

By a noncommutative Jordan ring is meant a ring in which the associative law is replaced by the weaker identities

$$
(x, y, x)=0 \text {, }
$$

and

$$
\left(x^{2}, y, x\right)=0
$$

where the associator $(a, b, c)$ is defined by $(a, b, c)=(a b) c-a(b c)$. A ring is flexible in case only (1.1) is assumed, and a ring is powerassociative provided

$$
x^{m} x^{n}=x^{m+n}
$$

holds in the ring for all positive integers $m, n$. It is known that a noncommutative Jordan ring of characteristic not 2 is power-associative [4], but there are flexible rings which are not power-associative. A ring $R$ is said to be of characteristic not 2 if $2 x=0$ implies $x=0$ in $R$.

2. Main results. 
THEOREM 2. Let $R$ be a power-associative ring and let $I$ be an ideal in $R$ such that $R / I$ is a finite division ring of characteristic $p>5$ with exactly $q$ elements. Suppose that (i) $I$ is commutative and every associator in the ideal generated by $I^{2}$ vanishes, and (ii) $x \equiv y(\bmod I)$ implies $x^{q}=y^{q}$ or both $x$ and $y$ commute with all elements of $I$. Then $R$ is commutative.

Proof. We first note that since $R / I$ is a finite power-associative division ring of characteristic $p>5, R / I$ is a finite field [1; Th. 5]. Hence for every $\bar{x} \in R / I, \bar{x}^{q}=\bar{x}$, whence for every $x \in R, x^{q} \equiv x(\bmod I)$. Now let $a_{0} \in I, b \in R$. We first wish to show that $a_{0} b=b a_{0}$. Suppose not. Let $a \in I$. Since $b+a \equiv b(\bmod I)$ and $a_{0} b \neq b a_{0}$, we have that

$$
(b+a)^{q}=b^{q} \text {. }
$$

Now by the power-associativity of $R$

$$
\left((b+a)^{q l},(b+a)^{q m},(b+a)\right)=0, \quad l, m \text { positive integers. }
$$

Hence, by $(2.1),\left(b^{q l}, b^{q m}, b+a\right)=0$, whence

$$
\left(b^{q l}, b^{q m}, a\right)=0 \text {. }
$$

Similarly

$$
\left(b^{q l}, a, b^{q m}\right)=\left(a, b^{q l}, b^{q m}\right)=0 .
$$

Since $b^{q l} \equiv b^{l}(\bmod I)$ and $b^{q m} \equiv b^{m}(\bmod I),(2.3)$ and the vanishing of every associator in the ideal generated by $I^{2}$ imply

$$
\left(b^{l}, b^{m}, a\right)=0 \text {. }
$$

Similarly, from (2.4),

$$
\left(a, b^{l}, b^{m}\right)=\left(b^{l}, a, b^{m}\right)=0 .
$$

We now show that the subring $R_{a_{0}, b}$ of $R$ generated by $a_{0}$ and $b$ is associative. It is sufficient to show that

$$
\left(\left\langle a_{0}, b\right\rangle_{1}\left\langle a_{0}, b\right\rangle_{2}\right)\left\langle a_{0}, b\right\rangle_{3}=\left\langle a_{0}, b\right\rangle_{1}\left(\left\langle a_{0}, b\right\rangle_{2}\left\langle a_{0}, b\right\rangle_{3}\right)
$$

where $\left\langle a_{0}, b\right\rangle_{i}$ denotes a finite product of $a_{0}$ 's and $b$ 's. If no $a_{0}$ appears in the left side of (2.7), then the equation holds by the power-associativity of $R$. If exactly one $a_{0}$ appears in the left side then it holds by either (2.5) or (2.6). Finally, if more then one $a_{0}$ appears in the left side, then (2.7) holds since every associator in the ideal generated by $I^{2}$ vanishes (also by (2.5) or (2.6) in some cases).

Now by the associativity of $R_{a_{0}, b}$ and the commutativity of $I$, we easily compute that 


$$
\left(a_{0} b+b\right)^{q}=b^{q}+a_{0} b^{q}+\sum_{i=1}^{q-1} b^{i} a_{0} b^{q-i}+\sum_{i=2}^{q}\left(\begin{array}{c}
q \\
i
\end{array}\right) a_{0}^{i} b^{q}
$$

and

$$
\left(b a_{0}+b\right)^{q}=b^{q}+b^{q} a_{0}+\sum_{i=1}^{q-1} b^{i} a_{0} b^{q-i}+\sum_{i=2}^{q}\left(\begin{array}{c}
q \\
i
\end{array}\right) a_{0}^{i} b^{q} .
$$

By the commutativity of $I$ and $a_{0} b \neq b a_{0},\left(a_{0} b+b\right) a_{0} \neq a_{0}\left(a_{0} b+b\right)$. Then, since $a_{0} b+b \equiv b a_{0}+b(\bmod I)$, we have by (ii) that

$$
0=\left(a_{0} b+b\right)^{q}-\left(b a_{0}+b\right)^{q}=a_{0} b^{q}-b^{q} a_{0} .
$$

Now $b^{q} \equiv b(\bmod I)$, hence by the commutativity of $I$

$$
a_{0} b^{q}-b^{q} a_{0}=a_{0} b-b a_{0} .
$$

But (2.8) and (2.9) imply that $a_{0} b=b a_{0}$, a contradiction. Hence $a b=b a$ for all $a \in I, b \in R$.

To complete the proof of the theorem, let $x, y \in R$. Since all elements of $I$ commute with all elements of $R$ we may assume that $x, y \notin I$. Since $R / I$ is a finite field, the multiplicative group of nonzero elements of $R / I$ is cyclic. Let $\bar{\xi}$ be a generator of this group and $\bar{\xi}=\xi+I, \xi \in R$. Then, for some integers $i, j$ and some $a_{1}, a_{2} \in I$, $x=\xi^{i}+a_{1}$ and $y=\xi^{j}+a_{2}$. By an easy computation we get $x y=y x$. Hence $R$ is commutative.

THEOREM 3. Let $R$ be a flexible power-associative ring and let $I$ be an ideal in $R$ such that $R / I$ is a finite division ring of characteristic $p>5$ with exactly $q$ elements. Suppose that (i) $I$ is commutative and associative, and (ii) $x \equiv y(\bmod I)$ implies $x^{q}=y^{q}$ or both $x$ and $y$ commute with all elements of $I$. Then $R$ is commutative.

Proof. Assume that $a_{0} b \neq b a_{0}$ for some $a_{0} \in I, b \in R$. Let $a \in I$ be arbitrary. We note that the proof of Theorem 2 is still valid through equation (2.4). Now since $b$ does not commute with all elements of $I$, we have by (ii), $x \equiv b(\bmod I)$ implies $x^{q}=b^{q}=\eta$ where $\eta$ is the common $q^{\text {th }}$ power of all the elements $x \equiv b(\bmod I)$. We observe that since $\eta^{q} \equiv \eta \equiv b^{q} \equiv b(\bmod I), \eta^{q}=b^{q}=\eta$. Equations (2.3) and (2.4) become

$$
\left(\eta^{l}, \eta^{m}, a\right)=\left(\eta^{l}, a, \eta^{m}\right)=\left(a, \eta^{l}, \eta^{m}\right)=0, \quad l>0, m>0 \text {, integers. }
$$

Now

$$
\left(\eta+a, \eta+a,(\eta+a)^{q l}\right)=0, \quad l>0 \text { an integer. }
$$

Since $\eta+a \equiv \eta(\bmod I),(\eta+a)^{q}=\eta^{q}=\eta$, hence by $(2.10)$, 
becomes

$$
\left(a, a, \eta^{l}\right)=0
$$

Similarly

$$
\left(a, \eta^{l}, a\right)=\left(\eta^{l}, a, a\right)=0 .
$$

Linearizing (2.12) and (2.13) we get

$$
\begin{aligned}
\left(a_{1}, a_{2}, \eta^{l}\right)+\left(a_{2}, a_{1}, \eta^{l}\right) & =\left(a_{1}, \eta^{l}, a_{2}\right)+\left(a_{2}, \eta^{l}, a_{1}\right) \\
& =\left(\eta^{l}, a_{1}, a_{2}\right)+\left(\eta^{l}, a_{2}, a_{1}\right) \\
& =0, \quad a_{1}, a_{2} \in I .
\end{aligned}
$$

We now wish to show by induction that

$$
\left(a^{m}, a, \eta^{l}\right)=\left(a, a^{m}, \eta^{l}\right)=0, \quad m>0, l>0 \text { integers. }
$$

For $m=1$ (2.15) is true by (2.12). Assume (2.15) is true for $m=k$. Now it is readily verified that in an arbitrary nonassociative ring, the Teichmüller identity holds:

$$
\begin{aligned}
0 & =h(w, x, y, z) \\
& =(w x, y, z)-(w, x y, z)+(w, x, y z)-w(x, y, z)-(w, x, y) z .
\end{aligned}
$$

Expanding $0=h\left(a, a^{k}, a, \eta^{l}\right)$ we obtain by induction and the associativity of $I$ that

$$
0=\left(a^{k+1}, a, \eta^{l}\right)-\left(a, a^{k+1}, \eta^{l}\right)
$$

whence by (2.14), $0=2\left(a^{k+1}, a, \eta^{l}\right)$. But then since $p \eta^{l} \in I$ and $I$ is associative,

$$
0=\left(a^{k+1}, a, p \eta^{l}\right)=p\left(a^{k+1}, a, \eta^{l}\right) .
$$

Therefore, since $p>2,0=\left(a^{k+1}, a, \eta^{l}\right)$. Hence (2.15) follows using (2.14).

Linearizing (1.1) yields

$$
0=(x, y, z)+(z, y, x) .
$$

Hence by (2.16), (2.15) yields

$$
\left(\eta^{l}, a, a^{m}\right)=\left(\eta^{l}, a^{m}, a\right)=0, \quad m>0, l>0 \text { integers. }
$$

The Jacobi identity

$$
(x y, z)+(y z, x)+(z x, y)=(x, y, z)+(y, z, x)+(z, x, y)
$$

can easily be shown to hold in an arbitrary nonassociative ring, where $(u, v)=u v-v u$. Now, in the Jacobi identity let $x=y=a, z=\eta^{l}$ 
and use (2.12), (2.13), and the commutativity of $I$ to obtain

$$
\left(a^{2}, \eta^{l}\right)=0 \text {. }
$$

We now show by induction that

$$
\left(a^{m}, \eta^{l}\right)=0, \quad m \geqq 2, l>0 \text { integers. }
$$

By (2.18), (2.19) is true for $m=2$. Assume (2.19) is true for $m=k$. Now, by (2.15), the induction assumption, the commutativity of $I$, and (2.17) we obtain

$$
a^{k+1} \eta^{l}=\left(a a^{k}\right) \eta^{l}=a\left(a^{k} \eta^{l}\right)=a\left(\eta^{l} a^{k}\right)=\left(\eta^{l} a^{k}\right) a=\eta^{l}\left(a^{k} a\right)=\eta^{l} a^{k+1} .
$$

Hence (2.19) follows.

By (2.15), (2.10) and (2.14), we easily obtain

$$
\left(a, a^{m} \eta^{n}, \eta^{l}\right)=\left(a^{m} \eta^{n}, a, \eta^{l}\right)=0, m>0, n>0, l>0 \text { integers. }
$$

Hence by (2.16)

$$
\left(\eta^{l}, a^{m} \eta^{n}, a\right)=\left(\eta^{l}, a, a^{m} \eta^{n}\right)=0, \quad m>0, n>0, l>0 \text { integers. }
$$

The anti-isomorphic copy $R^{\prime}$ or $R$ satisfies the hypotheses of the theorem, hence (2.20) and (2.21) hold in $R^{\prime}$. Therefore, in $R$, we have

$$
\begin{aligned}
0 & =\left(\eta^{l}, \eta^{n} a^{m}, a\right)=\left(\eta^{l}, a, \eta^{n} a^{m}\right) \\
& =\left(a, \eta^{n} a^{m}, \eta^{l}\right)=\left(\eta^{n} a^{m}, a, \eta^{l}\right), \quad m>0, n>0, l>0 \text { integers. }
\end{aligned}
$$

We wish to show by induction that

$$
(a \eta)^{m}=a^{m} \eta^{m}, \quad m>0 \text { an integer. }
$$

Indeed, using in order (2.20), the commutativity of $I,(2.15)$, and (2.10), we compute

$$
\left(a^{k} \eta^{k}\right)(a \eta)=\left(\left(a^{k} \eta^{k}\right) a\right) \eta=\left(a\left(a^{k} \eta^{k}\right)\right) \eta=\left(a^{k+1} \eta^{k}\right) \eta=a^{k+1} \eta^{k+1},
$$

hence the result. Considering the anti-isomorphic copy $R^{\prime}$ of $R$ again yields, because of (2.23),

$$
(\eta a)^{m}=\eta^{m} a^{m}, \quad m>0 \text { an integer. }
$$

Next we show by induction that

$$
(\eta+a)^{m}=\eta^{m}+\sum_{i=0}^{m-1}\left(\eta^{i} a\right) \eta^{m-i-1}+\sum_{i=2}^{m}\left(\begin{array}{c}
m \\
i
\end{array}\right) a^{i} \eta^{m-i}, \quad m \geqq 2,
$$

where by convention $\eta^{0} x=x \eta^{0}=x$ for all $x \in R$. Clearly this is true for $m=2$. Assume it is true for $m=k$. Then 


$$
\begin{aligned}
(\eta+a)^{k+1} & =\left(\eta^{k}+\sum_{i=0}^{k-1}\left(\eta^{i} a\right) \eta^{k-i-1}+\sum_{i=2}^{k}\left(\begin{array}{c}
k \\
i
\end{array}\right) a^{i} \eta^{k-i}\right)(\eta+a) \\
& =\eta^{k+1}+\sum_{i=0}^{k-1}\left(\left(\eta^{i} a\right) \eta^{k-i-1}\right) \eta+\sum_{i=2}^{k}\left(\begin{array}{c}
k \\
i
\end{array}\right)\left(a^{i} \eta^{k-i}\right) \eta \\
& +\eta^{k} a+\sum_{i=0}^{k-1}\left(\left(\eta^{i} a\right) \eta^{k-i-1}\right) a+\sum_{i=2}^{k}\left(\begin{array}{c}
k \\
i
\end{array}\right)\left(a^{i} \eta^{k-i}\right) a
\end{aligned}
$$

By (2.10),

$$
\left(\left(\eta^{i} a\right) \eta^{k-i-1}\right) \eta=\left(\eta^{i} a\right) \eta^{k-i}, \quad i=0, \cdots, k-1,
$$

and

$$
\left(a^{i} \eta^{k-i}\right) \eta=a^{i} \eta^{k+1-i}, \quad \mathrm{i}=2, \cdots, k .
$$

Next, using in order the commutativity of $I$, (2.22), commutativity of $I$ again, (2.13), (2.18), and (2.10), we compute for $i=0, \cdots, k-1$

$$
\begin{aligned}
\left(\left(\eta^{i} a\right) \eta^{k-i-1}\right) a & =a\left(\left(\eta^{i} a\right) \eta^{k-i-1}\right)=\left(a\left(\eta^{i} a\right)\right) \eta^{k-i-1}=\left(\left(\eta^{i} a\right) a\right) \eta^{k-i-1} \\
& =\left(\eta^{i} a^{2}\right) \eta^{k-i-1}=\left(a^{2} \eta^{i}\right) \eta^{k-i-1}=a^{2} \eta^{k-1}
\end{aligned}
$$

Finally, using the commutativity of $I$ and (2.15) we compute for $i=2, \cdots, k$

$$
\left(a^{i} \eta^{k-1}\right) a=a\left(a^{i} \eta^{k-i}\right)=a^{i+1} \eta^{k-i} .
$$

Therefore, (2.26) becomes

$$
\begin{aligned}
(\eta+a)^{k+1} & =\eta^{k+1}=\sum_{i=0}^{k-1}\left(\eta^{i} a\right) \eta^{k-i}+\sum_{i=2}^{k}\left(\begin{array}{c}
k \\
i
\end{array}\right) a^{i} \eta^{k+1-i} \\
& +\eta^{k} a+\sum_{i=0}^{k-1} a^{2} \eta^{k-1}+\sum_{i=2}^{k}\left(\begin{array}{c}
k \\
i
\end{array}\right) a^{i+1} \eta^{k-i} .
\end{aligned}
$$

Hence upon collecting terms we have

$$
(\eta+a)^{k+1}=\eta^{k+1}+\sum_{i=0}^{k}\left(\eta^{i} a\right) \eta^{k-i}+\sum_{i=2}^{k+1}\left(\begin{array}{c}
k+1 \\
i
\end{array}\right) a^{i} \eta^{k+1-i},
$$

which completes the proof of (2.25).

Now, in (2.25) replace $a$ by $a \eta$ and by $\eta a$ to obtain, respectively

$$
(\eta+a \eta)^{m}=\eta^{m}+\sum_{i=0}^{m-1}\left(\eta^{i}(a \eta)\right) \eta^{m-i-1}+\sum_{i=2}^{m}\left(\begin{array}{c}
m \\
i
\end{array}\right)(a \eta)^{i} \eta^{m-i},
$$

and

$$
(\eta+\eta a)^{m}=\eta^{m}+\sum_{i=0}^{m-1}\left(\eta^{i}(\eta a)\right) \eta^{m-i-1}+\sum_{i=2}^{m}\left(\begin{array}{c}
m \\
i
\end{array}\right)(\eta a)^{i} \eta^{m-i} .
$$

But by (2.19), (2.23), and (2.24), $(a \eta)^{i}=(\eta a)^{i}, \mathrm{i} \geqq 2$. Moreover, by (2.10) 


$$
\left(\eta^{i}(a \eta)\right) \eta^{m-i-1}=\left(\eta^{i} a\right) \eta^{m-i}
$$

and

$$
\left(\eta^{i}(\eta a)\right) \eta^{m-i-1}=\left(\eta^{i+1} a\right) \eta^{m-i-1}, \quad i=0, \cdots, m-1 .
$$

Therefore, upon subtracting (2.28) from (2.27) we obtain

$$
(\eta+a \eta)^{m}-(\eta+\eta a)^{m}=a \eta^{m}-\eta^{m} a .
$$

Now, since $\eta+a \eta \equiv \eta+\eta a \equiv b(\bmod I)$ and since $a_{0} b \neq b a_{0},(\eta+a \eta)^{q}=$ $(\eta+\eta a)^{q}$ which implies by (2.29) that $a \eta^{q}=\eta^{q} a$. However, since $b^{q}=\eta^{q}, b^{q}-b \in I$, and $I$ is commutative,

$$
\begin{aligned}
0 & =a_{0}\left(b-b^{q}\right)-\left(b-b^{q}\right) a_{0}=a_{0} b-b a_{0}-a_{0} b^{q}+b^{q} a_{0} \\
& =a_{0} b-b a_{0}-a_{0} \eta^{q}+\eta^{q} a_{0}=a_{0} b-b a_{0},
\end{aligned}
$$

a contradiction. Hence $a b=b a$ for all $a \in I, b \in R$.

The proof of Theorem 3 is completed exactly as that of Theorem 2 .

3. Remarks. The following example is a model for our theorems which is not associative. First, we define $R_{1}, I_{1}$ by

$$
\begin{aligned}
& R_{1}=\left\{\left(\begin{array}{ll}
\mathrm{a} & b \\
0 & 0
\end{array}\right) \mid a, b \in G F(p)\right\} ; \quad I_{1}=\left\{\left(\begin{array}{ll}
0 & b \\
0 & 0
\end{array}\right) \mid b \in G F(p)\right\} ; \\
& p>5 \text { a prime. }
\end{aligned}
$$

Now, let " $\bigcirc$ " denote the Jordan product in $R_{1}$ :

$$
\left(\begin{array}{ll}
a & b \\
0 & 0
\end{array}\right) \bigcirc\left(\begin{array}{ll}
c & d \\
0 & 0
\end{array}\right)=\left(\begin{array}{cc}
a c & \frac{a d+b c}{2} \\
0 & 0
\end{array}\right) .
$$

Since $p \neq 2, a d+b c / 2 \in G F(p)$. Let $R=R_{1}(+, \bigcirc)$, and let $I=I_{1}(+, \bigcirc)$. It is readily verified that $R$ is a Jordan ring which satisfies all the hypotheses of our theorems. Moreover, $R$ is not associative. Other models for our theorems appear in [2,3]. Also, examples are exhibited in those papers which show that the theorems fail in case $R$ fails to satisfy all of the hypotheses of these theorems.

It can be shown that for the proof of Theorem 2, the condition that every associator contained in the ideal generated by $I^{2}$ vanishes may be replaced by the more technical condition

$$
\left(x, a_{1}, a_{2}\right)=\left(a_{1}, x, a_{2}\right)=\left(a_{1}, a_{2}, x\right)=0 \text { for all } x \in R, a_{1}, a_{2} \in I .
$$

Finally, an examination of [1, Th. 5] and the proofs of our theorems will reveal that they also hold when the characteristic of $R / I$ is 3 or 5 providing the center of $R / I$ has more than five elements. 


\section{REFERENCES}

1. A. A. Albert, On nonassociative division algebras, Trans. Amer. Math Soc. 72 (1952), 296-309.

2. E. C. Johnsen, D. L. Outcalt, and Adil Yaqub, A commutativity theorem for noncommutative Jordan rings, Math. Jap. 11 (1967), 167-176.

3. D. L. Outcalt and Adil Yaqub, A generalization of Wedderburn's Theorem, Proc. Amer. Math. Soc. 18 (1967), 175-177.

4. R. D. Schafer, Noncommutative Jordan algebras of characteristic 0, Proc. Amer. Math. Soc. 6 (1955), 472-475.

Received October 3, 1967, and in revised form April 5, 1968. The first two authors were supported by Air Force Office of Scientific Research Grant AFOSR 698-67, and the third author by National Science Foundation Grant GP-5929.

University of California, Santa Barbara

UNIVERSITY OF HAWAII 


\section{PACIFIC JOURNAL OF MATHEMATICS}

\section{EDITORS}

\section{H. ROYDEN}

Stanford University

Stanford, California

\author{
R. R. Phelps \\ University of Washington \\ Seattle, Washington 98105
}

\section{J. DugundJI}

Department of Mathematics University of Southern California Los Angeles, California 90007

\section{RICHARD ARENS}

University of California

Los Angeles, California 90024

\section{ASSOCIATE EDITORS}

\section{E. F. BECKENBACH}

B. H. NEUMANN

F. WOLF

K. YOSIDA

\section{SUPPORTING INSTITUTIONS}

UNIVERSITY OF BRITISH COLUMBIA CALIFORNIA INSTITUTE OF TECHNOLOGY UNIVERSITY OF CALIFORNIA MONTANA STATE UNIVERSITY UNIVERSITY OF NEVADA NEW MEXICO STATE UNIVERSITY OREGON STATE UNIVERSITY UNIVERSITY OF OREGON OSAKA UNIVERSITY UNIVERSITY OF SOUTHERN CALIFORNIA

\author{
STANFORD UNIVERSITY \\ UNIVERSITY OF TOKYO \\ UNIVERSITY OF UTAH \\ WASHINGTON STATE UNIVERSITY \\ UNIVERSITY OF WASHINGTON \\ AMERICAN MATHEMATICAL SOCIETY \\ CHEVRON RESEARCH CORPORATION \\ TRW SYSTEMS
}

NAVAL WEAPONS CENTER

Mathematical papers intended for publication in the Pacific Journal of Mathematics should be in typed form or offset-reproduced, double spaced with large margins. Underline Greek letters in red, German in green, and script in blue. The first paragraph or two must be capable of being used separately as a synopsis of the entire paper. It should not contain references to the bibliography. Manuscripts, in duplicate if possible, may be sent to any one of the four editors. All other communications to the editors should be addressed to the managing editor, Richard Arens, University of California, Los Angeles, California 90024.

Each author of each article receives 50 reprints free of charge; additional copies may be obtained at cost in multiples of 50 .

The Pacific Journal of Mathematics is published monthly. Effective with Volume 16 the price per volume (3 numbers) is $\$ 8.00$; single issues, $\$ 3.00$. Special price for current issues to individual faculty members of supporting institutions and to individual members of the American Mathematical Society: $\$ 4.00$ per volume; single issues $\$ 1.50$. Back numbers are available.

Subscriptions, orders for back numbers, and changes of address should be sent to Pacific Journal of Mathematics, 103 Highland Boulevard, Berkeley 8, California.

Printed at Kokusai Bunken Insatsusha (International Academic Printing Co., Ltd.), 7-17, Fujimi 2-chome, Chiyoda-ku, Tokyo, Japan.

PUBLISHED BY PACIFIC JOURNAL OF MATHEMATICS, A NON-PROFIT CORPORATION

The Supporting Institutions listed above contribute to the cost of publication of this Journal, but they are not owners of publishers and have no responsibility for its content or policies. 


\section{Pacific Journal of Mathematics}

\section{Vol. 27, No. $2 \quad$ February, 1968}

Leonard E. Baum and George Roger Sell, Growth transformations for

functions on manifolds ............................ 211

Henry Gilbert Bray, A note on CLT groups ................... 229

Paul Robert Chernoff, Richard Anthony Rasala and William Charles

Waterhouse, The Stone-Weierstrass theorem for valuable fields....... 233

Douglas Napier Clark, On matrices associated with generalized

interpolation problems ................................

Richard Brian Darst and Euline Irwin Green, On a Radon-Nikodym theorem for finitely additive set functions . ...................... 255

Carl Louis DeVito, A note on Eberlein's theorem..................... 261

P. H. Doyle, III and John Gilbert Hocking, Proving that wild cells exist . . . 265

Leslie C. Glaser, Uncountably many almost polyhedral wild $(k-2)$-cells in

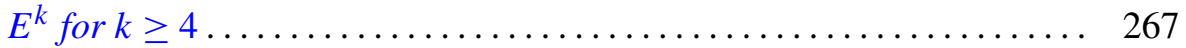

Samuel Irving Goldberg, Totally geodesic hypersurfaces of Kaehler

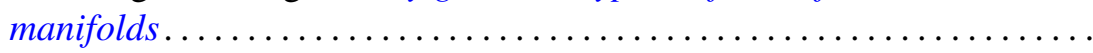

Donald Goldsmith, On the multiplicative properties of arithmetic functions .................................... 283

Jack D. Gray, Local analytic extensions of the resolvent ............ 305

Eugene Carlyle Johnsen, David Lewis Outcalt and Adil Mohamed Yaqub,

Commutativity theorems for nonassociative rings with a finite division ring homomorphic image ....................

André (Piotrowsky) De Korvin, Normal expectations in von Neumann

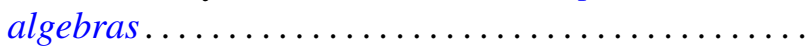

James Donald Kuelbs, A linear transformation theorem for analytic

Feynman integrals..........................

W. Kuich, Quasi-block-stochastic matrices ................... 353

Richard G. Levin, On commutative, nonpotent archimedean

semigroups ............................... 365

James R. McLaughlin, Functions represented by Rademacher series ... . . . 373

Calvin R. Putnam, Singular integrals and positive kernels............ 379

Harold G. Rutherford, II, Characterizing primes in some noncommutative

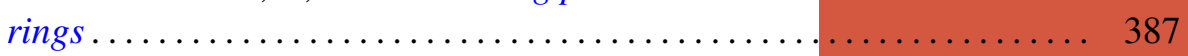

Benjamin L. Schwartz, On interchange graphs................... 393

Satish Shirali, On the Jordan structure of complex Banach *algebras . . . . . 397

Earl J. Taft, A counter-example to a fixed point conjecture............. 405

J. Roger Teller, On abelian pseudo lattice ordered groups ..... 